\title{
Indirect Translation: A Critical Study
}

H. LAKSHMI

\section{Abstract}

Despite the wide spread activity related to indirect translation, no theoretical body related to this activity, nor even a specific term to refer to it, exists in any of the Indian languages. This suggests that it is not considered as a practice that is different from direct translation and thus remains, as elsewhere, an under-researched, under-theorized area largely ignored in the rapidly evolving field of Translation Studies both as theory and practice. There is every need for research in this area since the role played by indirect translations not only in facilitating accessibility to texts that would otherwise remain inaccessible but also in fulfilling some function in the target context cannot be ignored by translation studies scholars. There is an urgent need to enlarge the field of Translation Studies by including indirect translation both as a process and product and to generate much needed body of theoretical knowledge related to it that would benefit both the translators and the translator trainers. The present paper is a humble beginning in this direction and an indirect translation made from Bengali into Telugu is taken up as a case study for studying the nature of this process.

Keywords: Indirect Translation, Filter Language, Mediating Language, Mahaswetha Devi, Bitter Soil, Salt.

An Indirect Translation (ITr), as understood from the term itself, is not a direct translation from a source text but an indirect one, i.e., a translation of a translation. It is a translation practice well-known and well-established all over the world 
and also has a long history. In the Indian context we are aware that the two epics - the Ramayana and the Mahabharat have been translated and retranslated several times over from other translations. In the present context, it is English and Hindi that have been serving as the mediating languages, facilitating translations from one Indian language into another. When it comes to translations from foreign languages, English has long been serving as the intermediary language. Despite the wide spread activity related to indirect translation, no theoretical body related to this activity, nor even a specific term to refer to it exists in any of the Indian languages, which suggests that it is not considered as a practice that is different from direct translation and thus remains, as elsewhere, an underresearched, under-theorized area largely ignored in the rapidly evolving field of Translation Studies both as theory and practice. It also suggests that it is being treated as a marginal activity, a necessary evil that need not be given importance in the field of Translation Studies.

It is perhaps this realization that prompted a conference on "Voice in Indirect Translation" held at the University of Lisbon (JET1 2013), and a panel presented at the 2013 congress of the European Society for Translation Studies, which eventually resulted in the publication of a special issue of Translation Studies (Vol.10, No.2, 2017) by Routledge. The inspiration for the present paper has also come from this special issue.

As is well known one apparent reason for why indirect translation takes place is the lack of competent translators between the given pair of languages. This is mainly the case for indirect translations that take place among Indian languages through English or Hindi as the intermediary language. Additionally "the difficulty of obtaining the original text"; or the difficulty in "translating from a geographically 


\section{H. Lakshmi}

and/or structurally distant languages" and "the higher price of translating from a distant language" are some of the motivating factors for ITr as mentioned by Alexandra Assis Rosa, Hanna Pieta\& Rita Bueno Maia (2017: 114).

Alexandra Assis Rosa, Hanna Pieta \& Rita Bueno Maia (2017) state that "Historically, ITr appears to decrease when adequacy or source-orientedness prevails, but increase when acceptability or target-orientedness prevails" (114). If we consider the Indian context in general, target orientedness prevailed until the translators came under the influence of western notions of translation and thus the distinction between the direct translation and indirect translation was never considered a matter of great significance. This is the reason why we do not have any term to refer to an indirect translation. Even in the case of acknowledged indirect translations made through a filter language, though the original source and its translation in the second language that served as the source to the present translation are acknowledged, but no other word except "Anuvaad" or "Anuvadam" is used to refer both to the activity and the resulting product. If we have to recognize this activity and make it visible, in the first place we have to think of a designated word to refer to this kind of indirect process of translation as it is the presence or absence of metalanguage that makes an activity visible or invisible. We can probably start using the self-explanatory terms, "pratyaksha anuvaad" and "paroksha anuvaad" indicating the division within the activity of translation and the latter need not be considered as something inferior to the former as each one has its own place and function in any given target polysystem in general.

Shibani Phukan (2003) seems to be the only one to refer to this directly. While comparing the western theories of translation with Indian theories of translation she points out the difference between the two stating that western theories of 
translation generally presume that translation is carried out directly from a source language into a target language but in India the practice of producing translations from translations, i.e., indirect translations, is fairly common.

In the present context, we can only assume that indirect translation in general is viewed negatively both by publishers and readers, especially the critics among them, as it is considered as something twice removed from the original. This could be the reason or one of the reasons why indirect translations are not always openly acknowledged as such. This remains only an assumption until and unless it is supported by concrete data.

There is every need for research in the area of indirect translation since the role played by it not only in facilitating accessibility to texts that would otherwise remain inaccessible but also in fulfilling some function in the target context is not something that can be ignored by translation studies scholars. Moreover, Translation Studies has moved further away from its earlier primary concern with the fidelity to the source text and literal versus free and such other binary notions, to other culturally more important issues after it has taken a cultural turn. In view of this, there is an urgent need to enlarge the field by including indirect translation both as process and product and to generate much needed body of theoretical knowledge related to it which would benefit both the translators and the translator trainers. The present paper is a humble beginning in this direction. An indirect translation made from Bengali into Telugu is taken up as a case study for studying the nature of this process and the issues involved in it.

At the outset, before moving on to the case study, I would like to raise certain questions that need to be addressed pertaining to indirect translation in the Indian context. We need to 
H. Lakshmi

ascertain data related to different cultural, historical, political and literary contexts in order to arrive at a comprehensive understanding of the following questions:

1. How common or natural indirect literary translations are in the Indian context?

2. Is there any historical account of the indirect translations in any of the Indian contexts?

3. What were the mediating or intermediary languages in the context of literary translations in India at different periods of history?

4. What are the language pairs that generally require intermediary translations?

5. What is the perception or attitude of the publishers and the reading public towards indirect translations?

6. What is the attitude of the government organizations that commission literary translations towards indirect translations?

7. Are indirect translations considered for awards/prizes on par with the direct translations?

8. Can the indirect translations be commissioned by any reputed agency?

9. Do the indirect translations remain overt or covert?

10.If they are overt is the identity of the intermediary translation, language and the translator of it acknowledged?

11.Is it possible to identify indirect translations and distinguish them from direct translations even if they are not acknowledged?

12. What are the consequences of the indirect translation from the perspective of the source language and the target 
language in terms of textual, cultural, conceptual and ideological aspects?

13.In terms of the translation theory and practice how does indirect translation differ from direct translation?

14. How does indirect translation impact translation pedagogy, CAT tools and even machine translation?

15.Like how literary texts are sometimes written keeping in mind their translatability into other languages can we also find translations produced in an international language or language of wider reach not meant for any direct readership but to facilitate further translations into various other languages of the world?

16. The last but not the least, is there any meta-discourse available regarding indirect translation in any of the Indian languages?

Coming to the case study, a comparative textual analysis of Mahaswetha Devi's short story 'Salt' and its Telugu translation-'Uppu' has been undertaken in this paper In order to understand the dynamics of indirect translation. This story is translated into Telugu not directly from Bengali original but its English translation by Ipsita Chanda, in the volume-Bitter Soil published by Seagull, Calcutta (first printing in 1998 and the second printing in 2002). Hyderabad Book Trust brought out an anthology of short stories by Mahasweta Devi, with the title "Rudhali" in Telugu in 1998. In this volume, two stories'Little ones' and 'Salt' were translated into Telugu from the above English translation. While the 'Little Ones' was translated by Achuth and 'Salt' was translated by Prabhanjan. Both have acknowledged the mediating translation in English and provided its details.

Here is the detailed textual analysis of the two texts-Salt in English and Uppu in Telugu: 
H. Lakshmi

1. NOT BY HAND, OR BY BREAD, nimak se marega- I'll kill you by salt, Uttamchand Bania had said.

చేత్తో కాదు.తిండితో కాదు . ఉప్పతో మిమ్మల్ని దెబ్బ కొడతా. ఉప్పతోనే మిమ్మల్ని చంపేస్తా అన్నాడు ఉత్తంచంద్.

(Back Translation (Hereafter BT): Not by hand, by food. I'll attack you by salt. I'll kill you by salt itself, said Uttamchand Bania).

The English translation and the Telugu translation respectively begin with the above lines. It can be noted from the above that the Telugu translation is a faithful reproduction of the English translation. It is faithful to the extent that even the double translation that is there in English, once in Hindi and then in English (namak se marega- I'll kill you by salt) is also retained, but both the sentences have been translated into Telugu, thus repeating the sentence, only emphasis is added to the second sentence. In the English translation, it is quite understandable that since the sentence in Hindi is retained, it needed to be translated into English. But in the Telugu translation when the sentence in Hindi is translated into Telugu, there is no need to give again the translation of its English translation. This unnecessary repetition could have been avoided had the Telugu translator understood the system of translation followed by the English translator. This illustration highlights an important point that needs the attention of the translators engaged in indirect translation, i.e., they have to first study the translation that they are taking as their source text and be clear about the method of translation adapted by the translator. This would help them overcome some of the issues involved in an indirect translation.

2. The village is bound in the shackles of betbegari to Uttanchand. 
ఆ ఉరు బేత్ బేగారి(వెట్టిచాకిరి) సంకెళ్తో ఉత్తంచంద్ గుప్పిట్లో ఉంది

(BT: The village is under the grip of Uttamchand with the shackles of beth begari (wageless labour).

3. What namak haraami!

ఎంత నమక్ హరామీలు (నమ్మక ద్రొహులు)

(BT: What namak haraamis (betrayers)!

4. Let them find out what ghato tastes like without salt. ఉప్పలేని ఘాటో(గంజి) రుచి ఎట్లా ఉంటుందో వాళ్కు తెలిసొస్తుంది.

(BT: They will come to know how ghato (gruel) tastes without salt).

5. This time Purti caught hold of the thikadar of the Forest Department- Give us work. Pay us in salt, not cash.

పూర్తి ఈసారి అటవీసాఖ ఠీకాదాద్(కాంట్రక్టర్) ను పట్టుకొని " మాకు పని ఇవ్వండి బాబూ! కూలికి పైసలివ్వనక్కర్లేదు, ఉప్పివ్వండి, చాలు!" అని ప్రాదేయ పడ్డాడు.

(BT: This time Purti caught hold of the thikadhadh (contractor) of the forest department and pleaded-"Give us work Sir! You do not have to pay us in cash, give us salt, that's all!")

6. They only eat ghato, maroa or boiled bhutta and vegetables or fruits or meat and fish.

వాళ్ళు వొట్టీ ఘూటొ, లేదా మారోవా (మొక్కజొన్న పిండి) లేదా ఉడికించిన భుట్టా (మక్కజొన్న ఖండె) ఇంకా వొట్టి కూరగాయలు, లేదా పళ్ళ, లేదా మాంసం, చేపలు తింటారు. 


\section{H. Lakshmi}

(BT: They only eat ghato, or maroa (maize flour) or boiled bhutta (corn ear) and plain vegetables, or fruits, or meat and fish.)

7. The film had mighty gunmen, gunfights, a voluptuous tongawalli and Amitabh Bachchan.

సినిమాలో గొప్ప గొప్ప తుపాకి దొంగలూ,ఒయ్యారం ఒలికించే ఒంపు సొంపుల టాంగావాలీ(గుర్రబ్బండి తోలే ఆమ్మాయి) అమితాబ్ బచన్ వగైరాలు ఉన్నారు.

(BT: The film had mighty robbers wielding guns, a voluptuous tongawali ( a woman driver of a horse-drawn cart), Amitabh Bachchan and the like.)

8. They knew that if the mahajan didn't lend them money, the junglee adivasis would die of starvation.

మహాజన్ అప్పివ్వకపోతే జంగ్లీ- (అడవి మనుషులైన ) ఆదివాసులు ఆకలితో చస్తారని వాళ్ళకు తెలుసు.

(BT: They knew that if the Mahajan does not lend them money, the junglee (uncultured people) adivasis would die of starvation.)

9. Finally, the daroga says- They must have been drunk.

చివరకు దరోగా (ఇన్ స్పెక్టర్) తేల్చేసాడు, " వాళ్ళు తప్పకుండా తప్పతాగి ఉంటారు" అని.

(BT: Finally, the daroga (Inspector) said, "they must have been drunk".)

Two points are to be made regarding the above sentences drawn from the texts under consideration. Firstly, in the 
English translation we find a glossary at the end where all the native words and expressions retained in the translation have been explained. But the Telugu translation, as can be observed from the above illustrations, gives the original Bengali words retained in the English translation and also a Telugu translation of their meaning given in the glossary in the English translation, in the text itself within parenthesis, next to the source word retained. This kind of double translation marks the foreignization that is resorted to in the translations. Even the English translation has followed the method of foreignization by retaining many native words and expressions used in the Bengali original, and it is more of a scholarly translation than a popular translation.

The Telugu translation as a result of the double translation becomes too clumsy and lacks readability. This kind of redundancy could have been avoided, had it been a direct translation. In the English translation it is necessary to explain all these native words and expressions and a glossary is thus added. The advantage of giving a glossary at the end is it does not hinder the readability of the text and helps only those readers who are keen on learning the cultural meanings and connotations of these source words. In the Telugu translation, by simply giving one translation, the one given within brackets, the translator would have made the text more readable, fluent and smooth. This is an issue that is likely to be common to any indirect translation from one Indian language into another made through English. If the intermediary translations were to be in Hindi this problem could have been avoided to a large extent. As translation studies scholars we are aware of the problems involved in using English in the Indian context as a medium for literary expression and exchange. All said and done it is a language not native to our soil and secondly any English knowing person from anywhere in the 


\section{H. Lakshmi}

globe can be a potential target reader for an English translation. It thus necessitates explanatory translations and footnotes and endnotes or glossaries which may not be required if the target readers are to be only Indians.

10. Across the breast of the sands, their figures gradually grow smaller.

ఇసుక తిన్నెలపై నడుస్తున్న ఝూఝు్ ఆదివాసుల ఆకారాలు క్రమంగా చిన్నవై పోయాయి.

(BT: The figures of the Jhujhur adivasis walking on the sand beds gradually grew smaller.)

11. At first, Purti and the others didn't give much importance to the unavailability of salt in the market.

అంగళ్ళలో తమకు ఉప్ప దొరకక పోవడాన్ని పూర్తి, ఇతర ఆదివాసులు మొదట్లో ఏమంత పట్టించుకోలేదు.

(BT: At first, Purti and other adivasis did not bother much about the unavailability of salt in the market.)

12. He wheedled and coaxed them into sending three boys from the village to school.

అతడు ఆదివాసులకు మంచి మాటలతో నచ్చ చెప్పాడు. ముగ్గురు పిల్లల్ని బడికి పంపడానికి వాళ్ళను

$$
\text { ఒప్పించాడు. }
$$

(BT: He convinced the adivasis with all his good talk. He made them agree to send three children to school.) 
Another point is observed in this analysis is related to the use of pronouns and nouns. As illustrated by the above examples, in most of the places where there is pronominal reference in English, the nouns are repeated in Telugu. While the pronouns they or them are used to refer to Adivasis in the English translation, the Telugu translation repeats the noun Adivasis itself, probably to make the text much more clear. The Bengali original has also, it seems, used pronouns just like the English translation rather than repeating the noun 'Adivasi'. Due to this reason, while the word Adivasi(s) is used only 7 times in the English translation, it has been used 27 times in the Telugu translation. This shift in the cohesive tie used in the text, from pronominal reference to lexical repetition, has also led to further added density to the text in Telugu. Additionally, this kind of repetition of the noun 'adivasis' in the Telugu translation so many times also has the impact of highlighting or emphasizing the fact that this is the story of adivasis or even representing them as 'the other'(similar to oriental representations that repeat the word 'Indians').

Another interesting point that has been observed in this study is related to the expression-'show business'. Consider the following:

The elephants of Betla understand 'show business'.

బేట్లా ప్రాంతపు ఏనుగులకు 'ఏో బిజినెస్ ' అర్థమవుతుంది.

(BT: The elephants of Betla understand "Show busisness".)

Here we can see that the expression- 'show business' has been retained in the Telugu translation. It is learnt that this is indeed the expression that is used in the original Bengali text. Here it needs to be pointed out that the Bengali text said to contain a lot of English words and some of them in the native pronunciation. The English translator has retained all the 


\section{H. Lakshmi}

English words and expressions that were used in the original Bengali and italicized them to indicate that they were actually given in English in the source text itself. In one case, even the native pronunciation of the word 'film' is indicated by writing the word as "fillum". Since the Telugu translator has no access to the Bengali source text and depended solely on the English translation this feature of the source text has gone completely missing in the Telugu translation. It is learnt from the English translator who is quite familiar with the variety of language spoken by the tribes represented in the story that the language spoken by these tribes is a mix of Bengali, Hindi and English; and a mix of class and caste registers. Unfortunately this point is not stated by the translator anywhere since the text has no translator's note or introduction. This point underscores the importance of the translator's note or introduction where certain aspects, both textual and contextual, related to the source text get highlighted and the strategies adapted by the translator would also be explained. This becomes crucial, even more, in the case of indirect translations where the second translator solely depends on the first translation. Fortunately, things are much better now as more and more publishers are encouraging the translators to come up with introduction and the translators themselves are insisting that their translation must go to print with an introduction.

\section{Conclusion}

The first point that is observed in this textual analysis is that, being a faithful reproduction of the English translation, the Telugu translation has retained all the original terms that have been retained in the English translation and whatever explanation that is provided in the glossary is also given within the text itself in parenthesis. The English translation since it is meant for both national and international audience needs to provide gloss to all the original terms and expressions that 
have been retained for the benefit of especially the non-Indian English readers. But if an Indian text is getting translated from one Indian language into another many of the cultural and religious terms and expressions need not be explained as they can be understood because of the shared culture. But then an indirect translation like the one under consideration faithfully tries to reproduce everything from the English translation without considering the fact that many of the explanations become redundant in a translation in an Indian language meant for Indian readership.

The second point I would like to highlight here is related to the modalities involved in a translation. I feel that even in an indirect translation the translator can cross check with the original Indian text rather than depending totally on the intermediary translation in English. In this context I suggest that collaborative translations would be a better option than indirect translations especially when the source language is an Indian language and the source text and optionally the source author is available for consultation. Many problems that come to the fore because of the indirect nature of the translation process, and more so when the intermediary translation is English, could be avoided by going for collaborative translations.

The third and the last point I would like to emphasize is that the translators engaged in indirect translation are required to analyze the translation that they take as their source text thoroughly in order to understand the translation strategy adapted by the translator and if possible, should get in touch with the translator, just like how a translator gets in touch with the source author if the latter is available and accessible for consultation. This would help reduce the gap between the source text and the indirect translation on the one hand and the influence of the intermediary translation on the other. 


\section{H. Lakshmi}

Translator's note, if available, would come to the rescue of the translator engaged in indirect translation in analyzing the intermediary translation and understanding the strategy/strategies adapted by its translator. The translator's awareness of the indirect nature of her/his translation and her/his acquaintance with translation theory would also go a long way in making the indirect translations not so indirect.

To conclude, there is a lot of scope for research in this area and lots of indirect translations are also available for analysis both from foreign languages into Indian languages and also from one Indian language into another. One can go for either synchronic studies or diachronic studies. Scholars can even take up collaborative projects to study this phenomenon and make useful contributions to both theory and practice of indirect translation.

\section{References}

Alexandra Assis Rosa, Hanna Pięta and Rita Bueno Maia. 2017. Theoretical, methodological and terminological issues regarding indirect translation: An overview, Translation Studies, $\quad 10: 2, \quad 113-132, \quad$ DOI: 10.1080/14781700.2017.1285247

Alvstad, Cecilia. 2017. Arguing for indirect translations in twenty-first-century Scandinavia. Translation Studies 10(2). 150-165. DOI: 10.1080/14781700.2017.1286254

Wikipedia.

Indirect

Translation.

https://en.wikipedia.org/wiki/Indirect_translation. Accessed on 5/8/2017

Devi, Mahasweta. 1998. Rudhaali Kathalu (Telugu). Hyderabad: Hyderabad Book Trust.

Devi, Mahasweta. 1998. Bitter soil. Stories by Mahasweta Devi. Translated into English by Ipsita Chanda. Calcutta: Seagull Books. 
Indirect Translation: A Critical Study

PhukAn, ShIBANI. 2003. Towards an Indian Theory of Translation. Wasafiri. 18(40). 27-30.

**** 